Bull. Mater. Sci., Vol. 20, No. 6, September 1997, pp. 879-883. (C) Printed in India.

\title{
Integrated computational chemistry system for the design of heterogeneous catalysts and nanostructured materials
}

\begin{abstract}
AKIRA MIYAMOTO
Department of Molecular Chemistry and Engineering, Faculty of Engineering, Tohoku University, Aoba-ku, Sendai 980-77, Japan

Abstract. The valuable catalytic and adsorptive properties of heterogeneous catalysts and the challenging area of nanostructure materials provide ample reason for establishing a firm theoretical understanding of their structure and behaviour. Computer simulation studies can contribute significantly in achieving an understanding of structure property relationships by the synthesis of current understanding and data, and by their capacity in revealing critical conceptual issues whose resolution demands additional experimentation. In the present communication I emphasize on the activity of our group in the area of computer simulation. Our group activity involves generation of new codes, better compatibility to solve the problem as well as application of the available computation technique in solving the problems generated in industry and in academics. We are concentrating on basic research as well as application using integrated computational chemistry as a tool.
\end{abstract}

Keywords. Computational chemistry system; application; overview.

\section{Introduction}

Computer-assisted molecular and materials design is a new area of chemistry which has been created by a significant advance in computer technologies and theoretical chemistry such as quantum chemistry, molecular dynamics, molecular mechanics, Monte Carlo simulation method, artificial intelligence, neural networks, data base, computer graphics, virtual reality and computational chemistry aided experimental measurements. Although the new methodology started from the drug design, i.e. the design of organic molecules, its impact has been widely spread to a variety of fields, including heterogeneous catalysts. It should also be emphasized that the methodology provides not only methods for scientific studies but also a key technology for the industrial innovations in research and development.

\section{Integrated computational chemistry system}

To contribute to such academic and industrial needs, we have been developing our own integrated computational chemistry system for the design of catalytic materials and inorganic functional materials which are composed of various softwares of quantum chemistry, molecular dynamics, Monte Carlo simulation, simulation of analytical methods, computer graphics and virtual reality coupled with different kinds of data base (figure 1). Quantum chemistry is effective for understanding electronic states of materials, nature of bonds and mechanism of chemical reactions. 


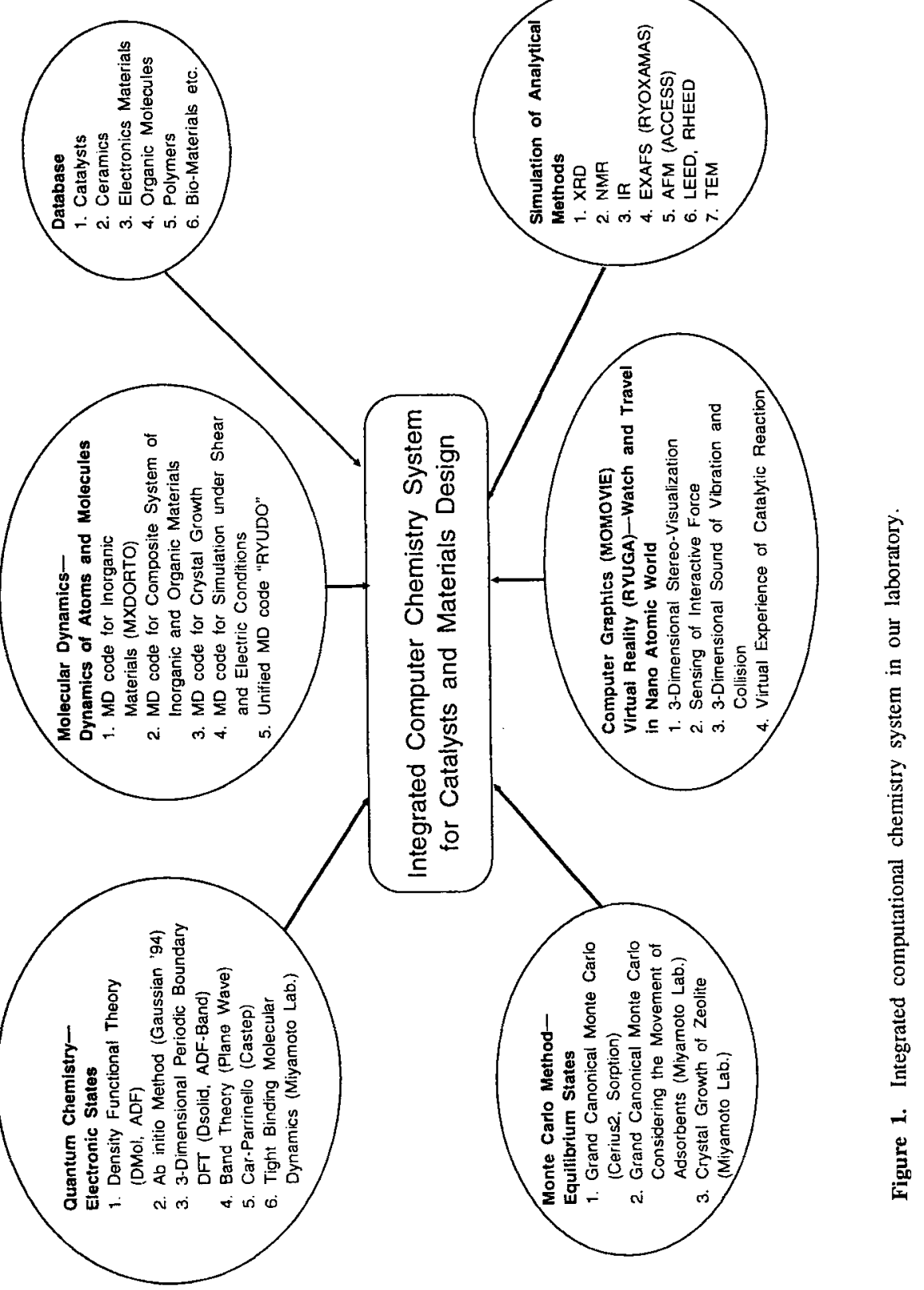


We have a variety of softwares of quantum chemistry in our system such as density functional codes (DMol, ADF), ab initio MO code (Gaussian '94), 3-dimensional periodic DFT codes (DSolid, ADF-Band), band calculation codes (Plane Wave), Car-Parrinello first principle molecular dynamics code (Castep) and tight-bonding molecular dynamics code developed in our laboratory. Molecular dynamics method is effective for understanding dynamics of atoms and molecules in materials. We have a variety of softwares of molecular dynamics in our system, including MXDORTO code for inorganic materials, a modified MXDORTO code for composite system of inorganic and organic materials, a modification of MXDORTO for crystal growth, another modification of MXDORTO for simulation of molecules under shear and electric conditions and RYUDO code developed in our laboratory. Monte Carlo simulation method is useful for understanding equilibrium states of systems. We have some softwares of Monte Carlo method in our system such as Grand Canonical Monte Carlo codes (Cerius2, Sorption), the same code developed in our laboratory allowing the movement of adsorbents, and a code of crystal growth of zeolites developed in our laboratory. Codes for the simulation of various analytical methods such as XRD, NMR, IR, EXAFS, AFM, LEED, RHEED and TEM are important parts of our system to have connections with real materials for experimental investigations. The RYOXAMAS code for EXAFS simulation and the ACCESS code for AFM simulation were developed in our laboratory. Various data base for structures and interatomic potentials are highly important for accumulating our trial-and-error experiences of computational chemistry and indispensable for a variety of applications of our system. In addition to the above methods and data base, we are greatly interested in human interfaces such as computer graphics and virtual reality and have developed MOMOVIE code for dynamic visualization and RYUGA code for virtual reality to watch and travel in nano atomic world.

\section{Various applications of the integrated computational chemistry system}

Most important policy of our group is to challenge applications of our integrated system to a variety of targets of industrial and academic importance (figure 2). We have a long experience in experimental work of heterogeneous catalyses including liquid metals, metal oxides, supported catalysts, zeolites, mixed oxide catalysts, metallosilicates, etc. Although previous catalytic technology has been based mainly on empirical approach, recent advance in surface analysis such as IR, ESR, AFM; STM, RHEED, LEED, NMR, TEM, etc enables us to clarify various phenomena in catalysis at atomistic level. This makes the application of computational chemistry attractive, because we can understand various interesting phenomena. The metalmicrocluster, nano-space, metal surface, chemical reaction on solid surface, excited states, heater-interface involved in heterogeneous catalysis provide most attractive and challenging topics for physics and chemistry, and the knowledge can widely be applied to a variety of fields such'as semiconductor, tribology, electronic device etc. On the basis of the above idea, we are interested in the applications of our computational methods to various topics of heterogeneous catalyses. We are also interested in the applications to adsorption and inorganic membrane technology, mechanical engineering and to the designing of electronic devices. 


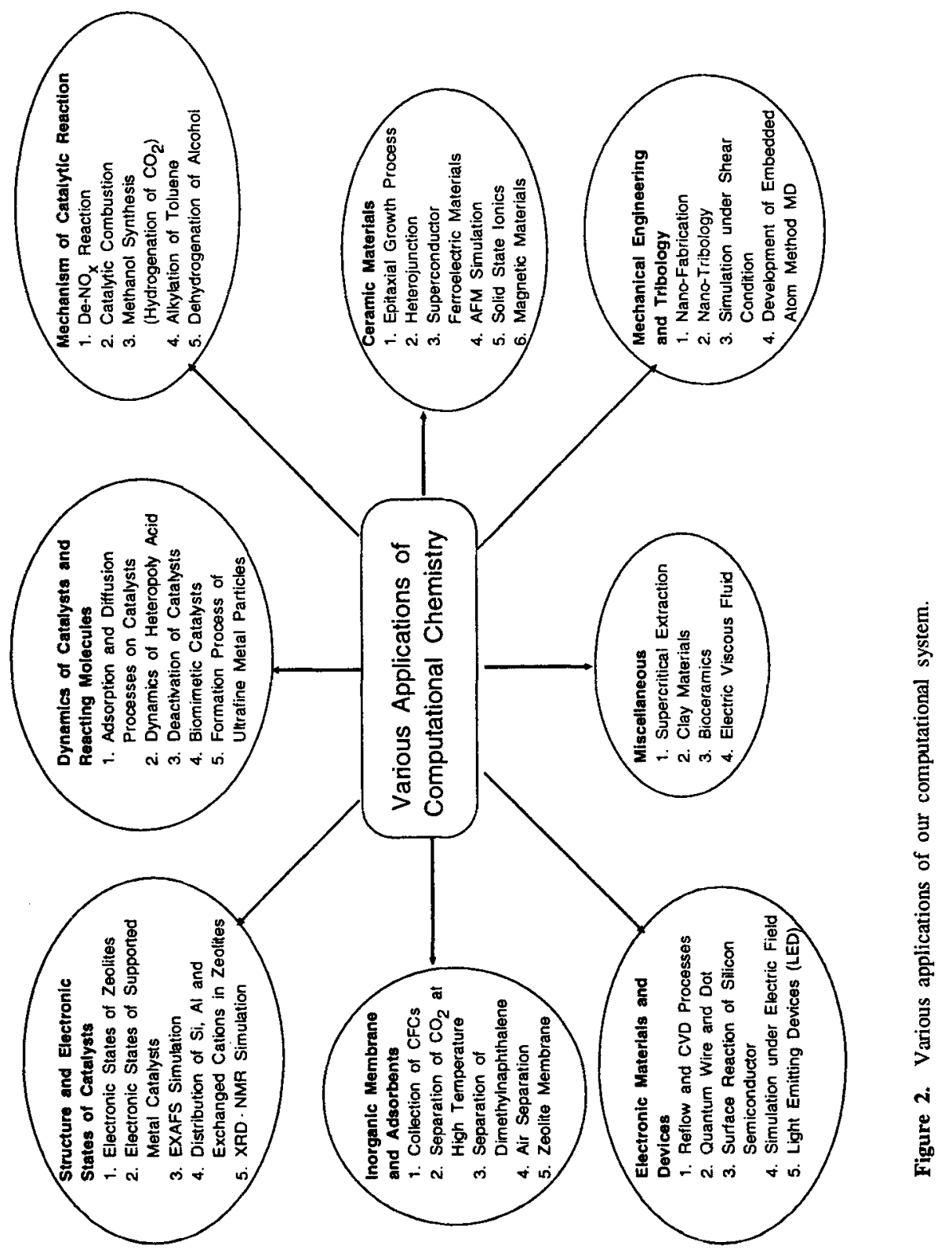


In the seminar, I tried to demonstrate that our integrated computational chemistry system is effective for investigating a variety of topics of industrial and academic importance as described below:

(I) Molecular dynamics approach to the structure of microporous materials.

(II) Grand Canonical Monte Carlo approach to the adsorption on zeolites.

(III) Combined use of molecular dynamics and quantum chemistry for the deNOx reaction on ion-exchanged ZSM-5.

(IV) Molecular sieving effect of carbon nanotubes.

(V) Inorganic membranes including zeolite membranes. 